\title{
HOLOMORPHIC MAPPINGS: SURVEY OF SOME RESULTS AND DISCUSSION OF OPEN PROBLEMS ${ }^{1}$
}

\author{
BY PHILLIP A. GRIFFITHS
}

1. Introduction. Our purpose is to survey some aspects of the global theory of holomorphic mappings, isolating along the way a few outstanding questions.

The general problem is this: Let $M$ and $N$ be complex manifolds and $f: M \rightarrow N$ a holomorphic mapping. Study the position of the image $f(M)$ in $N$. In particular, if $S \subset N$ is a complex analytic subvariety, then setting $S_{f}=f^{-1}(S)$, study the $S_{f} \subset M$ as $S$ varies among the subvarieties of $N$.

The most important special case of this problem is when $M=C^{m}$ and $N=\boldsymbol{P}^{n}$, the complex projective space. Then $f$ may be given by $n$ entire meromorphic functions

$$
f(z)=\left(f_{1}(z), \ldots, f_{n}(z)\right), \quad z=\left(z_{1}, \ldots, z_{m}\right) \in C^{m} .
$$

The subvarieties $S$ will be the zero sets of polynomials $p_{\alpha}(w)$ ( $w$ $\left.=\left(w_{1}, \ldots, w_{n}\right) \in C^{n}\right)$, and so our question amounts to globally studying solutions of the equations

$$
\begin{aligned}
& w_{j}=f_{j}(z) \quad\left(z \in C^{m}\right), \\
& p_{\alpha}(w)=0 .
\end{aligned}
$$

The following two examples illustrate the extremes in what is understood about this problem.

EXAmPle 1. Suppose that $f: \boldsymbol{C} \rightarrow \boldsymbol{P}^{1}$ is an entire meromorphic function, so that (1) reduces to studying the roots of the equation

$$
f(z)=a, \quad z \in C \text { and } a \in \boldsymbol{P}^{1} .
$$

The most immediate global property is the Liouville theorem, which says that the image $f(\boldsymbol{C})$ is dense in $\boldsymbol{P}^{1}$, unless of course $f$ is constant. A much more precise result is the Picard theorem, which states that a nonconstant $f$ can omit at most two values. Finally, the most penetrating study of the equation (2) is that by $R$. Nevanlinna [2], who found that, with at most two exceptional values $a \in \boldsymbol{P}^{1}$, the "density" of the solutions of (2) in the disc $|z|<r$ is positive as $r \rightarrow \infty$. This result is a beautiful and far reaching quantitative refinement of the Picard theorem, and will be discussed further below.

EXAMPLE 2. Fatou and Bieberbach found a holomorphic mapping $f: \boldsymbol{C}^{2} \rightarrow \boldsymbol{P}^{2}$ which is one-to-one and whose image omits an open set in $\boldsymbol{P}^{2}$.

1 Talk presented at the dedication of "Surge L," Stonybrook, New York, October 16. 1971. Printed by invitation of the editors; received October 28, 1971. 
Thus, the Liouville property and its subsequent refinements fail completely. To illustrate further how little we understand, Mark Green pointed out to me that it seems to be unknown whether the Euclidean volume of the image of an entire holomorphic mapping $f: C^{2} \rightarrow C^{2}$ is necessarily infinite!

To see in principle how Fatou-Bieberbach mappings can arise, we let $V_{0} \subset C^{2}$ be an analytically embedded finite Riemann surface. Thus $V_{0}$ is a compact Riemann surface minus at least one disc of positive radius, and as such there exists a bounded holomorphic function $h_{0}$ on $V_{0}$. It is easy to construct such $V_{0}$ in $C^{2}$, and this Riemann surface is then given by an equation

$$
g(x, y)=0
$$

where $g \in \mathcal{O}\left(C^{2}\right)$ is an entire holomorphic function on $C^{2}$. Moreover, there is $h \in \mathcal{O}\left(C^{2}\right)$ which restricts to $h_{0}$ on $V_{0}$. Now we assume the following stability property: Setting $V_{u}=\{(x, y): g(x, y)=u\}$ and $h_{u}=h \mid V_{u}$, the functions $h_{u}$ are bounded for all $|u|<\varepsilon$. If this is satisfied, then the map

$$
f=(g, h): C^{2} \rightarrow P^{2}
$$

omits an open neighborhood of $(0, \infty)$. The Fatou-Bieberbach example is of this general character.

These examples illustrate the fact that the equations (1) will have a much different behaviour when $\operatorname{codim}(S)=1$ than when $\operatorname{codim}(S)>1$. In the former case, there is pretty good evidence that the Picard theorem and Nevanlinna theory should go through more or less in general, and this will be discussed in the next two sections. In the latter case, the hope is that a reasonable theory can be expected for certain maps $f$, and this will be discussed in $\$ 4$ below. In $\$ 5$ we return to the value distribution theory of divisors in a more algebro-geometric setting.

2. Picard-type theorems for divisors. We want to discuss the qualitative position of the image for a holomorphic mapping

$$
f: \boldsymbol{C}^{\boldsymbol{m}} \rightarrow \boldsymbol{P}^{n}
$$

relative to the divisors on $\boldsymbol{P}^{n}$. The most important divisors are linear hyperplanes, but it is essential to also study the case of hypersurfaces of any degree. Indeed, the understanding of this latter situation should not be too much different from that of a holomorphic mapping into a general smooth, projective variety $V$ (cf. Carlson's thesis [6]).

We begin with some notations. A (positive) divisor $D$ in $\boldsymbol{P}^{n}$ may be uniquely written as a sum

$$
D=n_{1} D_{1}+\cdots+n_{l} D_{l}
$$


where the $D_{j}$ are distinct irreducible hypersurfaces of degree $d_{j}$ and $n_{j}>0$. Then the degree of $D$ is

$$
\operatorname{deg}(D)=n_{1} d_{1}+\cdots+n_{l} d_{l} .
$$

We shall say that $D$ has normal crossings if (i) all the $D_{j}$ are smooth and all the multiplicities $n_{j}=1$; and (ii) the local defining equations for the $D_{j}$ passing through a fixed point always form part of a holomorphic coordinate system. In case the $D_{j}$ are linear hyperplanes, this means exactly that $D_{1}, \ldots, D_{l}$ are in general position. We denote the number $l$ of components of $D$ by \#(D).

Let $|D|$ be the complete linear system of all divisors of a fixed degree $d$. Any such $D$ is given by the zeroes of a homogeneous polynomial $P$ of degree $d$, and $P$ is unique up to nonzero multiples. Thus $|D|$ is a projective space. Furthermore, the Fubini-Study metric on $\boldsymbol{P}^{n}$ induces a metric on $|D|$, and we may let $d \mu(D)$ be the associated volume form normalized so that

$$
\int_{D \in|D|} d \mu(D)=1
$$

We are now ready to discuss a holomorphic mapping (3). The usual Liouville theorem immediately implies that for nonconstant $f$ the image $f\left(C^{m}\right)$ meets a dense set of divisors in $|D|$ for any fixed degree. Indeed, for any two divisors $D_{1}, D_{2} \in|D|$ there is a meromorphic function $\phi$ on $\boldsymbol{P}^{n}$ such that $\phi=0$ on $D_{1}$ and $\phi=\infty$ on $D_{2}$. If the image $f\left(C^{m}\right)$ omits a neighborhood of $D_{2}$ in $|D|$, then all pull-backs $\phi \circ f$ are bounded holomorphic functions on $\boldsymbol{C}^{\boldsymbol{m}}$. Corresponding to the Picard theorem, the principal results are the following:

(5) TheORem (M. GReEN [5]). Let $f: \boldsymbol{C}^{m} \rightarrow \boldsymbol{P}^{n}$ be a holomorphic mapping whose image omits $n+k$ linear hyperplanes in general position. Then the image is contained in a projective linear subspace of dimension $[n / k]$, and this bound is sharp.

Corollary 1. A nondegenerate equi-dimensional map $f: \boldsymbol{C}^{n} \rightarrow \boldsymbol{P}^{n}$ can omit at most $n+1$ hyperplanes in general position.

Corollary 2. A nonconstant holomorphic curve $f: C \rightarrow P^{n}$ can omit at most $2 n$ hyperplanes in general position.

Observe that both of these corollaries reduce to the usual Picard theorem when $n=1$. Somewhat weaker versions of these results appear in Wu's book [3], and on the basis of these he was led to conjecture both of the corollaries. Similar results to the above have also been obtained by Fujimoto.

(6) THEOREM ([6] AND [8]). An equi-dimensional holomorphic mapping 
$f: \boldsymbol{C}^{n} \rightarrow \boldsymbol{P}^{n}$ which omits a divisor $D$ with normal crossings and $\operatorname{deg}(D) \geqq n+2$ must be degenerate.

In case $D$ is a linear combination of hyperplanes, this theorem reduces to Corollary 1 , above. In contrast to Corollary 2 , the following example due to Kiernan shows that we cannot force a holomorphic curve to be degenerate simply by omitting a smooth hypersurface of arbitrarily high degree.

Example 3. Let $\varepsilon=(-1)^{1 / n}$. Then the map $t \rightarrow[t, \varepsilon t, 1]$ from $C$ to $\boldsymbol{P}^{2}$ omits the smooth curve $X_{0}^{n}+X_{1}^{n}+X_{2}^{n}=0$ for any $n>0$.

Problem A. Let $D$ be a divisor in $\boldsymbol{P}^{n}$ with normal crossings. Then, under what conditions on the degree $\operatorname{deg}(D)$ and number of components \#(D) is a holomorphic mapping $f: \boldsymbol{C}^{\boldsymbol{m}} \rightarrow \boldsymbol{P}^{n}-D$ necessarily constant?

Problem B. Let $D$ be a divisor in $\boldsymbol{P}^{n}$ with normal crossings and $f: C \rightarrow \boldsymbol{P}^{n}-D$ a holomorphic curve which misses $D$. Then if $\operatorname{deg}(D)$ $\geqq n+2$, does the image $f(\boldsymbol{C})$ lie in an algebraic subvariety of $\boldsymbol{P}^{n}$ ?

3. Nevanlinna theory for divisors. We continue with the notations of $\$ 2$. Let $f: \boldsymbol{C}^{\boldsymbol{m}} \rightarrow \boldsymbol{P}^{n}$ be a holomorphic mapping and set $D_{f}=f^{-1}(D)$ for a divisor $\boldsymbol{D}$ in $\boldsymbol{P}^{n}$. Nevanlinna theory is a quantitative study of the "size" of $D_{f}$ as $D$ varies in a complete linear system $|D|$. To make this precise, we shall use the following notations:

$$
\begin{aligned}
B[r] & =\left\{z \in C^{m}:\|z\| \leqq r\right\}=\text { ball of radius } r \text { in } C^{m} \\
D_{f}[r] & =D_{f} \cap B[r] \\
\omega & =\frac{(-1)^{1 / 2}}{2 \pi}\left\{\sum_{j=1}^{m} d z_{j} \wedge d \bar{z}_{j}\right\}=\text { flat Kähler form on } C^{m} ; \\
v(D, r) & =\int_{D_{f}[r]} \omega^{n-1}=\text { area of } D_{f}[r] ; \\
N(D, r) & =\int_{0}^{r} v(D, t) d t / t^{2 m-1}=\text { counting function; and } . \\
T(r) & =\int_{D \in|D|} N(D, r) d \mu(D)=\text { order function. }
\end{aligned}
$$

Thus, $N(D, r)$ measures the area of $D_{f}$ in the ball of radius $r$, and $T(r)$ is the average of the counting functions over all divisors of a fixed degree. The first main theorem (F.M.T.) of Nevanlinna theory [8] has as corollary the following remarkable inequality (Nevanlinna inequality):

$$
N(D, r)<T(r)+O(1) .
$$

Geometrically, this says that the size of any particular $D_{f}$ is always bounded 
by the average size over all divisors in the linear system. (Here we make the obvious assumption that $f\left(C^{m}\right)$ does not lie in any $D$.) Using (7), we may introduce the Nevanlinna defect

$$
\delta(D)=1-\varlimsup_{r \rightarrow \infty}[N(D, r) / T(r)],
$$

which has the two properties:

$$
\begin{aligned}
0 & \leqq \delta(D) \leqq 1, \quad \text { and } \\
\delta(D) & =1 \quad \text { if } f\left(C^{m}\right) \text { does not meet } D .
\end{aligned}
$$

In general, if $\delta(D)>0$, then the image of $f$ meets $D$ less often than on the average. Integration of (7) leads to the relation

$$
\int_{D \in|D|} \delta(D) d \mu(D)=0,
$$

which is a strong version of the Liouville theorem.

The principal result in general Nevanlinna theory is the Ahlfors defect relation [3]:

(10)THEOREM. Let $f: \boldsymbol{C} \rightarrow \boldsymbol{P}^{n}$ be a holomorphic curve which does not lie in a hyperplane, and suppose that $D_{1}, \ldots, D_{l}$ are hyperplanes in general position. Then

$$
\sum_{j=1}^{l} \delta\left(D_{j}\right) \leqq n+1 .
$$

For $n=1$, this is the famous defect relation of R. Nevanlinna. However, the $n$-dimensional version is by no means a direct generalization of the R. Nevanlinna result. The most trivial consequence of (10) is that a nondegenerate holomorphic curve can omit at most $n+1$ hyperplanes in general position. This $n$-dimensional version of the Picard theorem, due to E. Borel, is also a corollary of Green's theorem [5]. The defect relation (10) is more subtle than either the R. Nevanlinna or E. Borel theorems, as a glance at the difficulty of the proof suggests.

There is also an equi-dimensional defect relation which deals with hypersurfaces rather than just hyperplanes.

(11) THEOREM [8]. Let $f: \boldsymbol{C}^{n} \rightarrow \boldsymbol{P}^{n}$ be a nondegenerate holomorphic mapping and $D_{1}, \ldots, D_{l}$ smooth divisors of degree $d$ such that $D=D_{1}+\cdots+D_{l}$ has normal crossings. Then

$$
\sum_{j=1}^{l} \delta\left(D_{j}\right) \leqq\left(\frac{n+1}{d}\right) .
$$

We observe that this result contains the R. Nevanlinna defect relation as 
well as Corollary 1 to Theorem (5). It is, however, less deep than the Ahlfors relation (10). A principal open question in the study of how the divisors in $\boldsymbol{P}^{n}$ meet the image of a holomorphic mapping $f: \boldsymbol{C}^{m} \rightarrow \boldsymbol{P}^{n}$ is

Problem C. Let $f: \boldsymbol{C}^{m} \rightarrow \boldsymbol{P}^{n}$ be a holomorphic mapping. Let $D_{1}, \ldots, D_{l}$ be smooth divisors in $|D|$, the complete linear system of divisors of degree $d$ in $\boldsymbol{P}^{n}$, and suppose that $D_{1}+\cdots+D_{l}$ has normal crossings. Assume finally that $f\left(C^{m}\right)$ does not lie in any $D \in|D|$. Then is it true that $\sum_{j=1}^{l} \delta\left(D_{j}\right)$ $\leqq((n+1) / d)$ ?

4. The case of higher codimension. Since the state of our knowledge is so primitive, we shall restrict our attention to the equi-dimensional case $f: \boldsymbol{C}^{n} \rightarrow \boldsymbol{P}^{n}$, assume that $f$ is everywhere nondegenerate, and ask how many linear subspaces of fixed dimension $d$ in $\boldsymbol{P}^{n}$ the image $f\left(\boldsymbol{C}^{n}\right)$ meets? Because of the Fatou-Bieberbach example, this is probably not a good question as it stands. It was Chern who first showed that maps satisfying certain growth conditions had a dense image in $\boldsymbol{P}^{n}$. The geometric nature of these "good" maps was clarified considerably by $\mathrm{Wu}$ [7], and Stoll put the theory in a general setting [4]. The answer which emerges is the following: Let $\omega$ be the flat Kähler form on $C^{n}, \phi$ the Kähler form of the Fubini-Study metric on $\boldsymbol{P}^{n}$, and $\phi_{f}=f^{*}(\phi)$. Define the order functions

$$
T_{k}(r)=\int_{0}^{r}\left\{\int_{B[t]} \phi_{f}^{k} \wedge \omega^{n-k}\right\} \frac{d t}{t^{2 n-2 k+1}},
$$

for $k=1, \ldots, n$. Then $T_{1}(r)$ is the order function used in $\$ 3$ above, and $T_{n}(r)$ measures the volume of the image $f\left(C^{n}\right)$. In general, the various $T_{k}(r)$ need have little relation to one another, which reflects the fact that writing $f=\left(f_{1}, \ldots, f_{n}\right)$ as an $n$-tuple of meromorphic functions, the various $f_{i}$ may not interact at all. Let $T_{k}^{\prime}(r)$ denote the derivative of $T_{k}(r)$.

(12) THEOREM. If $\lim _{r \rightarrow \infty}\left[T_{k-1}^{\prime}(r) / T_{k}(r)\right]=0$, then the image $f\left(C^{n}\right)$ meets almost all linear subspaces of codimension $k$ in $\boldsymbol{P}^{n}$.

For $k=1$, this says that $f$ should be nonconstant. If $f$ is uniformly quasi-conformal in the sense that, for unit tangent vectors $\tau_{1}, \tau_{2}$ to $C^{n}$,

$$
\left[\sup _{\tau_{1}} \phi_{f}\left(\tau_{1}\right)\right] /\left[\inf _{\tau_{2}} \phi_{f}\left(\tau_{2}\right)\right] \leqq c<\infty,
$$

then Theorem (12) holds $(\mathrm{Wu})$. Such maps therefore satisfy the Liouville property relative to the linear subspaces of any dimension in $\boldsymbol{P}^{n}$.

A phenomenon related to the Fatou-Bieberbach example is the failure of the Nevanlinna inequality (7) for higher codimension: Let $h \in \mathcal{O}\left(C^{n}\right)$ be an entire holomorphic function, $D=\{h(z)=0\}$ the zero set of $h$, and

$$
M_{h}(r)=\max _{\|z\| \leqq r} \log |h(z)|
$$


the logarithm of the maximum modulus of $h$. Then the inequality (7) has as an easy consequence the estimate

$$
v(D, r) / r^{2 n-2} \leqq c M_{h}(2 r),
$$

which bounds the growth of the area of $D$ in terms of the modulus of $h$. On the other hand, recent work by Shiffman and Cornalba shows that there exist entire functions $h_{1}, h_{2} \in \mathcal{O}\left(C^{2}\right)$ such that the number of common zeroes

$$
\begin{aligned}
h_{1}(z) & =0, \\
h_{2}(z) & =0, \\
\|z\| & \leqq r
\end{aligned}
$$

in the ball of radius $r$ cannot be estimated by $M_{h_{1}}(\lambda r)$ and $M_{h_{2}}(\lambda r)$ for any $\lambda>0$.

In conclusion, the examples together with existing results perhaps suggest the following: There should be a subclass of the holomorphic mappings $f: \boldsymbol{C}^{m} \rightarrow \boldsymbol{P}^{n}$ which have good geometric and analytic properties. Such maps should satisfy a Nevanlinna inequality (7) and the Liouville property (9).

Moreover, there should be functorial properties, such as: The restriction $f \mid \boldsymbol{C}^{k} \rightarrow \boldsymbol{P}^{n}$ belongs to the class if $f: \boldsymbol{C}^{m} \rightarrow \boldsymbol{P}^{n}$ does. This class of maps, if it exists, would be the place where a reasonable geometric study of entire holomorphic maps takes place.

The situation is perhaps a little reminiscent of studying the equation $f(z)=a$ where $f(z)$ is a meromorphic function defined only in a finite disc $|z|<R$. Then the value distribution theory has a good meaning only for those maps which satisfy $T(r) \rightarrow \infty$ as $r \rightarrow R$, where $T(r)$ is the Nevanlinna characteristic function (cf. [2]).

5. Value distribution theory for divisors on general algebraic varieties. In this section we assume some knowledge of algebraic geometry, line bundles (especially the canonical bundle), etc. A good reference is Chern [1].

Referring to Corollary 2 of Theorem (5) and Theorem (10), the integer $n+1$ has special meaning for $\boldsymbol{P}^{n}$, because a sum $D_{1}+\cdots+D_{n+1}$ of $n+1$ hyperplanes is in the anti-canonical series $\left|K^{*}\right|$ where $K$ is the canonical line bundle on $\boldsymbol{P}^{n}$. This observation leads to a generalization of the equidimensional defect relation (11). To state this, we let $V$ be a smooth, projective variety of dimension $n$ with canonical bundle $K_{V}$. For a divisor $D$ on $V,[D]$ denotes the corresponding line bundle, and for any line bundle $L$, $c_{1}(L)$ is the first Chern class. If $\alpha, \beta \in H^{2}(V, R)$ are cohomology classes and $\lambda \in \boldsymbol{R}$ is a real number, then $\lambda>[\alpha / \beta]$ means that $\lambda \beta-\alpha \in H^{2}(V, \boldsymbol{R})$ is 
represented by a positive definite $(1,1)$ form. Similarly, we may define $\hat{\lambda} \leqq[\alpha / \beta]$.

(14) THEOREM [8]. Let $L$ be a positive line bundle and $D_{1}, \ldots, D_{l} \in|L|$ smooth divisors such that $D=D_{1}+\cdots+D_{l}$ has normal crossings. Suppose that $f: C^{n} \rightarrow V$ is a nondegenerate holomorphic mapping, and define Nevanlinna defects $\delta(D)(D \in|L|)$ by (8). Then

$$
\sum_{j=1}^{l} \delta\left(D_{j}\right) \leqq\left[c_{1}\left(K_{V}^{*}\right) / c_{1}(L)\right] .
$$

COROLLARY. If the canonical bundle $K_{V}$ is positive, then any holomorphic mapping $f: C^{n} \rightarrow V$ is degenerate.

When $V=\boldsymbol{P}^{n}$, this result reduces to Theorem (11) above. What is obviously desirable is to have a defect relation in the nonequidimensional case, even, or perhaps most importantly, for a holomorphic curve $f: C \rightarrow V$. For this, the analogue of Problem $\mathrm{C}$ is the following:

Problem D. With the notations of Theorem (14) above, suppose that $f: C \rightarrow V$ is a holomorphic curve such that the image $f(C)$ does not lie in a divisor $D \in\left|L+K_{V}\right|$. Then is $\sum_{j=1}^{l} \delta\left(D_{j}\right) \leqq\left[c_{1}\left(K_{V}^{*}\right) / c_{1}(L)\right]$ ?

Two special cases of this problem occur when $K_{V}>0$ and $K_{V}=0$. In the first case, we may take $L$ to be trivial and, specializing to the case $\operatorname{dim} V=2$, we have the

Problem E. Let $V$ be an algebraic surface with positive canonical bundle and $f: C \rightarrow V$ a holomorphic mapping. Then does the image $f(C)$ lie in an algebraic curve in $V$ ?

One difficulty in this question is that, contrary to the equi-dimensional case, $f$ may be a highly transcendental mapping and still have image contained in algebraic curve. Since, for a given line $\boldsymbol{P}^{1}$ in $\boldsymbol{P}^{3}$ there are smooth surfaces of arbitrarily high degree containing $\boldsymbol{P}^{1}$, problem $\mathrm{E}$ represents a best possible conclusion. It is not known whether or not there are a finite number of rational maps $f: C \rightarrow V$ having distinct images; and this is another difficulty in the problem.

In case $K_{V}=0$, we are led to the following question:

Problem F. Let $V$ be an abelian variety, $f: C \rightarrow V$ a holomorphic mapping, and $D$ a positive divisor on $V$. Then does the image $f(C)$ meet $D$ ? In case $f$ is a group homomorphism, this problem (and more) was done by $\mathbf{J}$. Ax in response to a question of Lang.

Another open question concerning holomorphic maps and complex tori is this :

Problem G. Let $V$ be an $n$-dimensional complex torus and $f: C^{n} \rightarrow V$ a nondegenerate holomorphic mapping. Then, is the image $f\left(C^{n}\right)$ dense in $V$ ?

Our conclusion may be taken as stating that the geometric position of holomorphic curves in general algebric varieties is understood very little. 


\section{BIBLIOGRAPHY}

The basic reference for complex manifolds and holomorphic mappings is

1. S. S. Chern, Complex manifolds without potential theory, Van Nostrand Math. Studies, no. 15, Van Nostrand, Princeton, N.J., 1967. MR 37 \#940.

This book also contains a short discussion of holomorphic curves and the defect relation of R. Nevanlinna. Further material on the classical value distribution theory is given in

2. R. Nevanlinna, Analytic functions, Springer-Verlag, Berlin and New York, 1970.

The Ahlfors theorem is given in

3. $\mathrm{H}$. Wu, The equidistribution theory of holomorphic curves, Princeton Univ. Press, Princeton, N.J., 1970.

A discussion of value distribution theory and first main theorems in a very general setting is given in

4. W. Stoll, Value distribution of holomorphic maps into compact complex manifolds, Lecture Notes in Math., no. 135, Springer-Verlag, Berlin and New York, 1970.

Picard-type theorems relative to linear hyperplanes in $\boldsymbol{P}^{n}$ are contained in

5. M. Green, Holomorphic maps into complex projective space omitting hyperplanes, Trans. Amer. Math. Soc. (to appear). (Preprints available from Princeton University, Princeton, N.J.)

An algebro-geometric approach to Picard theorems is given in

6. J. Carlson, Some degeneracy theorems for entire functions with values in an algebraic variety, Trans. Amer. Math. Soc. (to appear). (Preprints available from Princeton University, Princeton, N.J.) in

The results of Chern and Wu on the value distribution theory of points in $P^{n}$ are presented

7. $\mathrm{H} . \mathrm{Wu}$, Remarks on the first main theorem of equidistribution theory. I, II, III, J. Differential Geometry 2 (1968), 197-202; ibid. 3 (1969), 83-94, 369-384.

A modern treatment for the Nevanlinna theory of divisors in the equidimensional case is given in

8. J. Carlson and P. Griffiths, $A$ defect relation for equidimensional holomorphic mappings between algebraic varieties, Ann. of Math. (Notes available from Princeton University, Princeton, N.J.)

Department of Mathematics, Princeton University, Princeton, New Jersey 08540 